\title{
Stellar masses calibrated with micro-lensed quasars
}

\author{
Paul L. Schechter ${ }^{1}$, Jeffrey A. Blackburne ${ }^{2}$, David Pooley ${ }^{3}$ and \\ Joachim Wambsganss ${ }^{4}$ \\ ${ }^{1}$ MIT Kavli Institute Cambridge, MA 02139, USA \\ email: schech@mit.edu \\ 2 Areté Associates, Northridge, CA 91324 \\ ${ }^{3}$ Sam Houston State University, Huntsville, TX 77341 \\ ${ }^{4}$ Zentrum für Astronomie der Universität Heidelberg, Germany
}

\begin{abstract}
We measure the stellar mass surface densities of early type galaxies by observing the micro-lensing of macro-lensed quasars caused by individual stars, including stellar remnants, brown dwarfs and red dwarfs too faint to produce photometric or spectroscopic signatures. Our method measures the graininess of the gravitational potential, in contrast to methods that decompose a smooth total gravitational potential into two smooth components, one stellar and one dark. We find the median likelihood value for the calibration factor $\mathcal{F}$ by which Salpeter stellar masses (with a low mass cutoff of $0.1 M_{\odot}$ ) must be multiplied is 1.23 , with a one sigma confidence range of $0.77<\mathcal{F}<2.10$.
\end{abstract}

Keywords. galaxies: stellar content, gravitational lensing

\section{Introduction}

Stellar masses for early type galaxies are almost always determined by one of two methods: either they are estimated from spectra (and sometimes only broad band colors) or they are deduced by subtracting the contribution of an assumed dark matter component from a combined mass inferred from kinematic (and sometimes macro-lensing) measurements. Multiple examples of both methods can be found in the present volume. Both methods have shortcomings.

Here we use a third method: determining the stellar mass surface density of an early type galaxy from brightness fluctuations of the four images of a background quasar that is both multiply-imaged ("macro-lensed") by the galaxy and "micro-lensed" by the individual stars in that galaxy (Schechter \& Wambsganss 2004; Kochanek 2004). This method, in contrast to spectral methods, is sensitive to stellar mass near and below the hydrogen burning limit, as well as to the mass in stellar remnants. And where dark matter subtraction methods make asumptions about the dark matter profiles, the gravitational micro-lensing technique makes only an assumption about the combined gravitational potential, one that has been subjected to extensive observational verification.

Ideally one would observe a single system long enough to see a great many fluctuations and infer an accurate stellar surface density. But the timescale for micro-lensing variations is of order ten years for a lens at redshift $z \sim 0.5$ (Mosquera \& Kochanek 2011), and observations of even four quasar images at a single epoch give only broad constraints on the mass surface density. So instead we observe ten systems at a single epoch and combine results. 

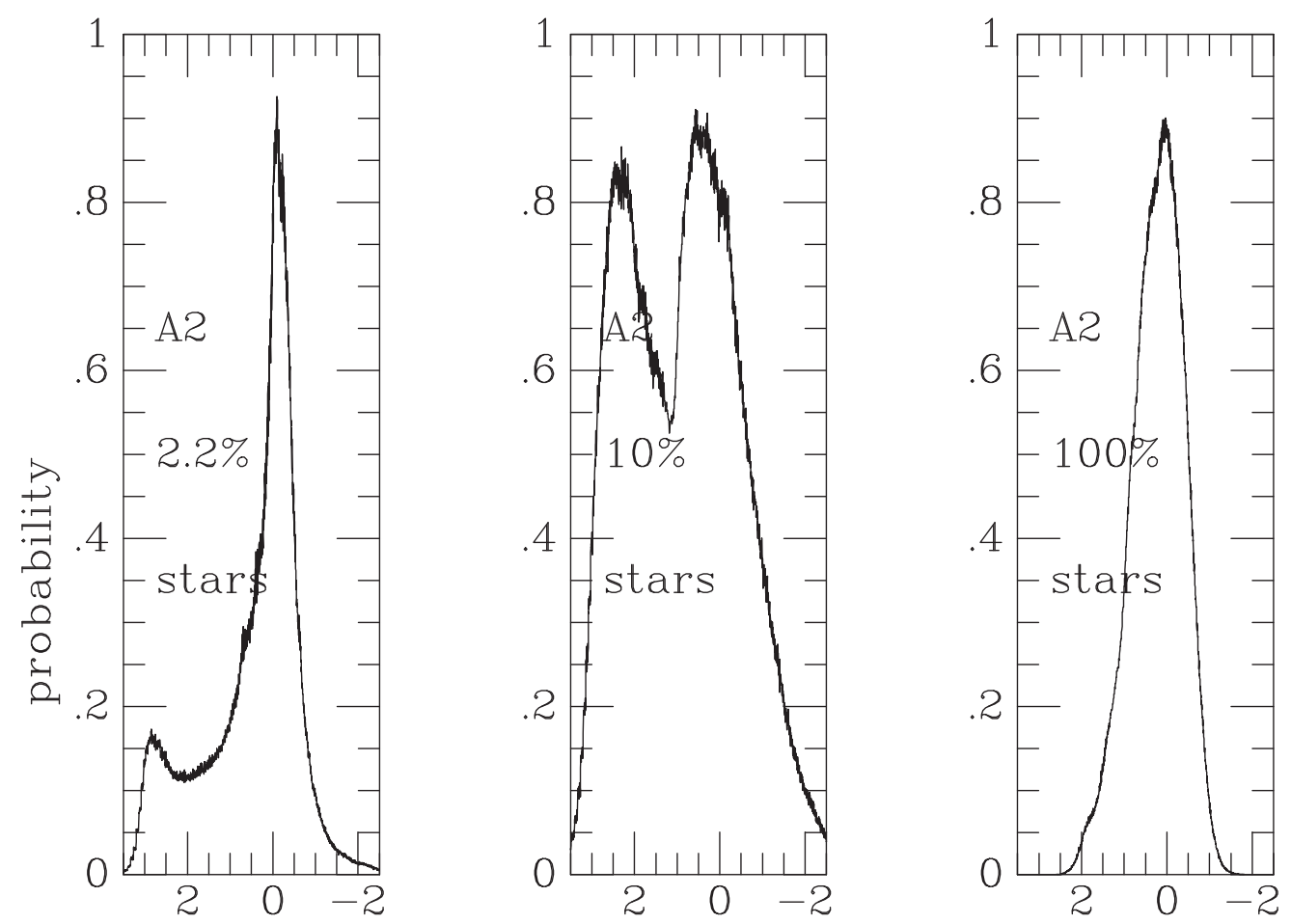

\section{magnitude difference}

Figure 1. Probability distribution for the ratio of observed to macro-model flux (expressed as a magnitude difference) for the $A 2$ image of the quadruple lens PG $1115+080$ for three different stellar mass fractions. The different shapes of the distributions permit determination of the stellar mass fraction.

\section{Calibrating the stellar mass fundamental plane}

Our approach is to use the stellar mass fundamental plane (Hyde \& Bernardi 2009) to predict the stellar surface mass densities at the positions of our quasar images, which cause differences between the observed quasar fluxes and those predicted by a macromodel. We then adjust the stellar masses by a multiplicative constant, $\mathcal{F}$. The constant is varied and the likelihood of the observed quasar X-ray fluxes is computed for each value of $\mathcal{F}$.

Our stellar mass fundamental plane was constructed using data from Auger et al. (2010) and Sonnenfeld et al. (2013), who used a Salpeter initial mass function (IMF) with a low mass cutoff of $0.1 M_{\odot}$ to compute stellar masses for lensing galaxies at $z \sim 0.2$ and $z \sim 0.5$ respectively. The median likelihood value of the factor by which these masses must be multiplied is $\mathcal{F}=1.23$ with a $68 \%$ confidence interval of $0.77<\mathcal{F}<2.10$. The range is the result of small sample size.

\section{Salient features of the analysis}

- We take the quasar to be point-like at X-ray wavelengths (relative to the Einstein rings of the micro-lensing stars) and use Chandra fluxes taken from Pooley et al. (2007). Blackburne et al. (2011) have shown that the optical and near-IR emitting regions are comparable in projected size to the stellar Einstein rings, rendering optical wavelengths less well suited to the present analysis. 


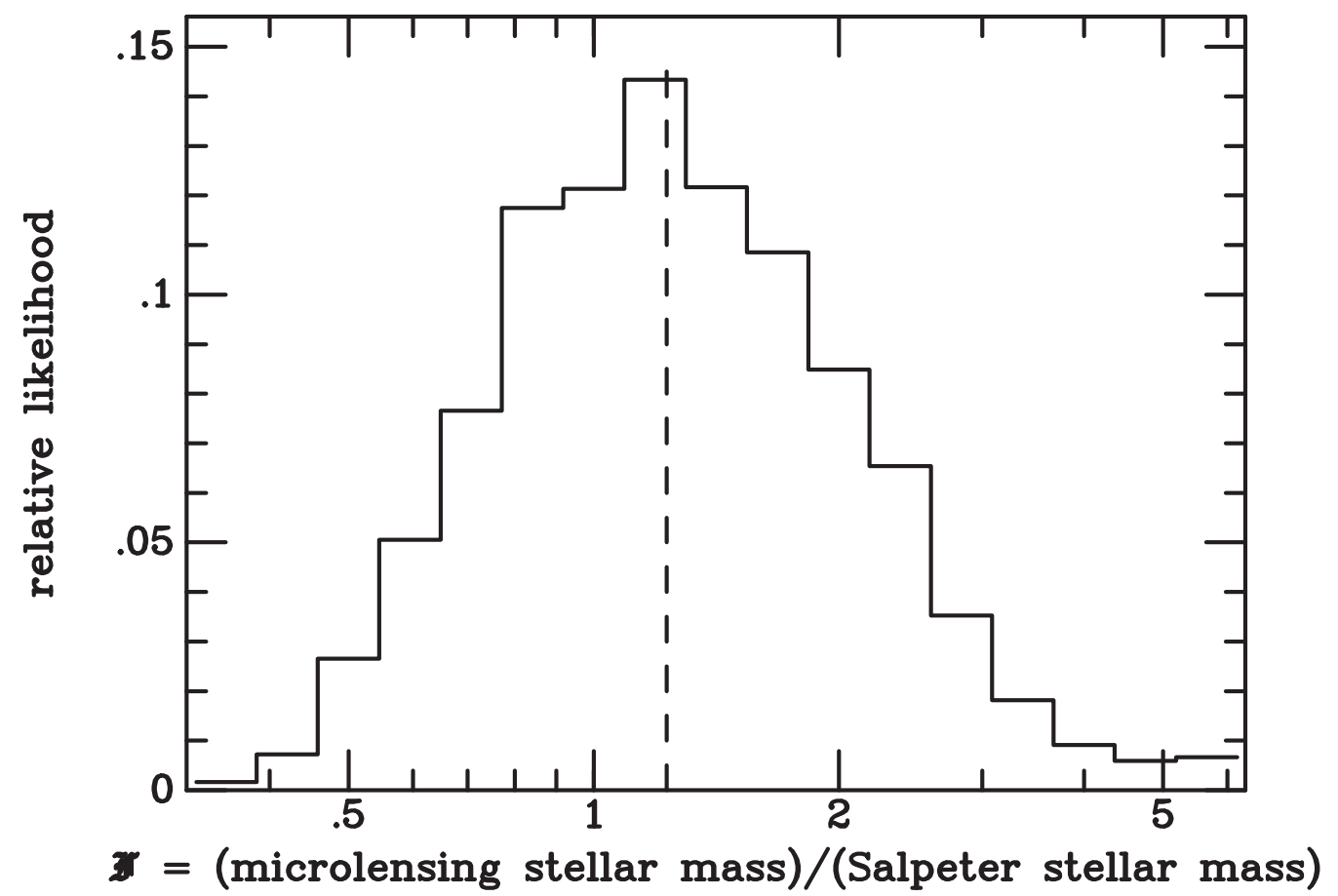

Figure 2. The likelihood histogram for the calibration factor $\mathcal{F}$ by which the stellar mass fundamental plane computed using a Salpeter IMF must be multiplied to produce the observed $\mathrm{X}$-ray flux ratio anomalies.

- We use a fundamental plane in which a "proxy" velocity dispersion computed from the radius of the Einstein ring of the macro-model is substituted for stellar dispersions. We find that for the Auger et al. (2010) sample this gives considerably less scatter than using proper stellar velocity dispersions in constructing the fundamental plane.

- We model the lenses as singular isothermal ellipsoids (ellipticity and orientation identical to that of the stellar light) with an external shear.

- We have analyzed the sensistivity of our result to a number of different possible sources of systematic error. We find them to be small compared to the statistical confidence interval. In particular we find that milli-lensing by dark matter substructure has very little effect on our result.

- Our result is most sensitive to the measured de Vaucouleurs radii of the lensing galaxies. The published values fall into two groups, based on the software used, which deviate systematically from each other; we adopt the geometric mean.

- An alternative analysis that takes surface mass density and effective radius to be functions of the proxy velocity dispersion - a "fundamental line" - circumvents the systematic uncertainties in the measured effective radii and yields a calibration factor $\mathcal{F}$ that differs by $7 \%$.

- The X-ray fluxes for the images of RX J0911+0551 pull our calibration factor $\mathcal{F}$ to higher values. The macro-models predict that image $b$, a minimum of the light travel time, should be more than five times brighter than image $d$, also a minimum, but is observed to be only $25 \%$ brighter.

- For a galaxy with a proxy velocity dispersion of $266 \mathrm{~km} / \mathrm{s}$ and an effective radius of $6.17 \mathrm{kpc}$, average for the Auger et al. (2010) sample, the calibrated fractional stellar 


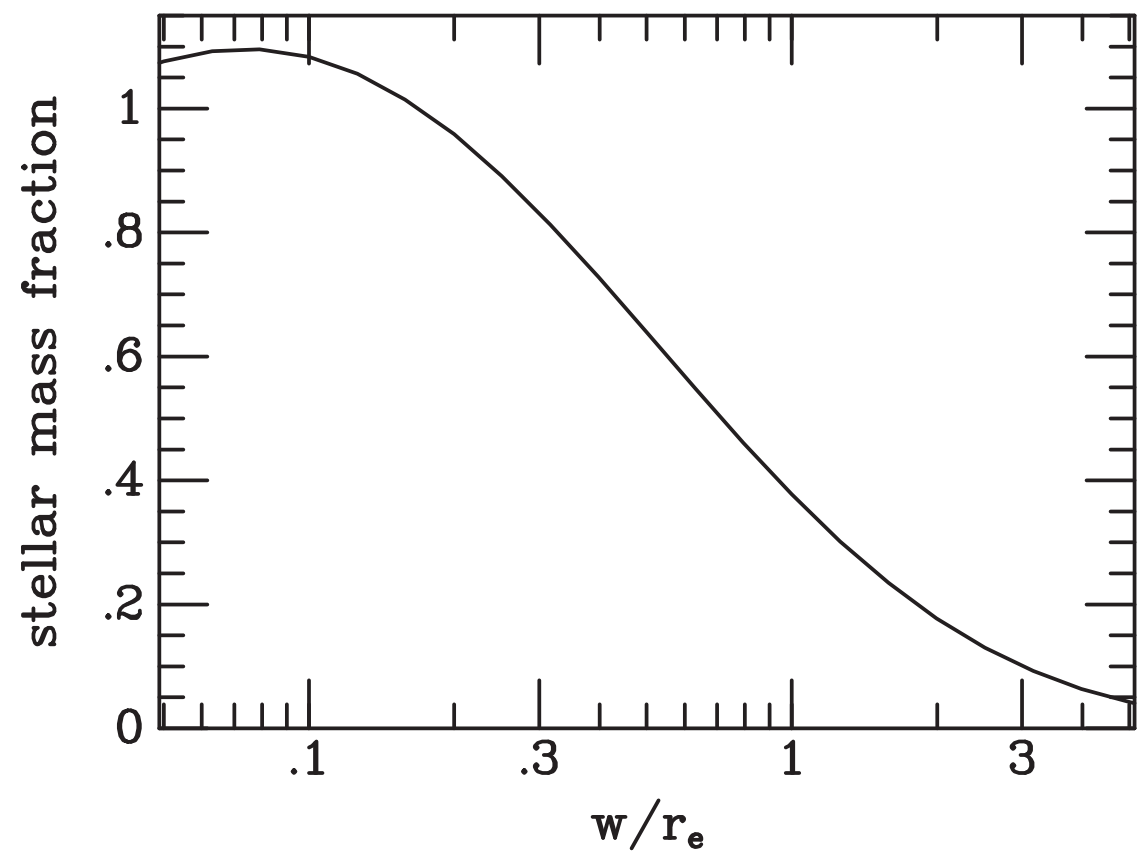

Figure 3. The stellar surface mass density as a fraction of the total for the typical lensing galaxy, obtained by applying the calibration factor $\mathcal{F}$ to the stellar mass fundamental plane derived from the SLACS + SL2S samples. The peak at $w / r_{e}=0.074$ (where $w$ is the circularized radius) occurs where the slope of the de Vaucouleurs profile is equal to that of the singular isothermal.

surface mass density peaks at a value of 1.10 at $0.074 r_{e}$, as shown in Figure 3 . At $1.5 r_{e}$, the typical Einstein radius for our lens systems, the stellar mass fraction is 0.25 .

The work summarized here is the culmination of an effort begun by Schechter \& Wambsganss (2004) and described in the proceedings of IAU Symposium 220. A more thorough description of the present analysis can be found in Schechter et al. (2014)

\section{Acknowledgements}

The authors gratefully acknowledge the very substantial contributions of Saul A. Rappaport. This work was supported in part by the US National Science Foundation under grants AST02-06010 and AST06-07601 and by NASA under Chandra grant G07-8099. The first author thanks the members of the organizing committee for their good efforts.

\section{References}

Auger, M. W., Treu, T., Bolton, A. S., et al. 2010, ApJ, 724, 511

Bate, N. F., Floyd, D. J. E., Webster, R. L., \& Wyithe, J. S. B. 2011, ApJ, 731, 71

Blackburne, J. A., Pooley, D., Rappaport, S., \& Schechter, P. L. 2011, ApJ, 729, 34

Hyde, J. B. \& Bernardi, M. 2009, MNRAS, 396, 1171

Kochanek, C. S. 2004, ApJ, 605, 58

Mosquera, A. M. \& Kochanek, C. S. 2011, ApJ, 738, 96

Pooley, D., Blackburne, J. A., Rappaport, S., \& Schechter, P. L. 2007, ApJ, 661, 19

Schechter, P. L., Pooley, D., Blackburne, J. A., \& Wambsganss, J. 2014, arXiv:1405.0038

Schechter, P. L. \& Wambsganss, J. 2004, in IAU Symposium, Vol. 220, Dark Matter in Galaxies, ed. S. Ryder, D. Pisano, M. Walker, \& K. Freeman, 103.

Sonnenfeld, A., Gavazzi, R., Suyu, S. H., Treu, T., \& Marshall, P. J. 2013, ApJ, 777, 97 